


\title{
La valorisation de la recherche : une nouvelle mission pour l'université?
}

\author{
Chantale Mailhot et Patrick Pelletier \\ HEC Montréal, Canada \\ Véronique Schaeffer \\ BETA, Université Louis Pasteur, Strasbourg, France
}

\section{RÉSUMÉ}

La valorisation des résultats de la recherche universitaire est un phénomène qui s'est accentué depuis quelques années et s'est accompagné d'importants changements institutionnels. Dans une économie de la connaissance, l'université se positionne comme un acteur socioéconomique central. Elle se préoccupe beaucoup d'organiser le transfert technologique et de développer une gestion de la propriété intellectuelle, courant le risque de réduire l'idée de valorisation de la recherche à la commercialisation de ses résultats. La question de la place et du rôle des universités dans la société aujourd'hui est l'objet de vifs débats pour les politiciens, les chercheurs universitaires et les industriels. Comment la valorisation, lorsqu'elle est vue comme une nouvelle mission de l'université, s'inscrit-elle à travers les changements de contexte et s'accommode-t-elle des missions traditionnelles d'enseignement et de recherche? Nous considérerons cette question en portant une attention particulière aux contextes canadien et québécois.

\begin{abstract}
Over the last few years the process of adding value to academic research results has become a growing phenomenon entailing important institutional changes. In the context of the knowledge economy, universities are considered to be central socio-economic actors. They are concerned
\end{abstract}


with the development and the organization of technology transfer and the management of intellectual property. However, the value-adding process runs the risk of becoming confined to the commercialization of research results. The evolution of the role of the university in the economy and society is a subject of debates among politicians as well as academic and industrial actors. What is the impact of the process of adding value to research, considered as a new mission of the university, on the changing academic environment and on the traditional role of the university? To study this question we focus on the cases of Canada and Quebec.

\section{INTRODUCTION}

Le phénomène de la valorisation des résultats de la recherche universitaire semble s'être accentué depuis quelques années. De plus en plus d'organisations, qu'elles soient à vocation politique, économique ou sociale, consacrent des ressources importantes aux activités touchant à la production des connaissances, à leur organisation et à leur diffusion. Tel est le cas de l'université qui, plus que jamais marquée du sceau de l'entrepreneurship universitaire, doit aujourd'hui se préoccuper des questions de transfert technologique et de gestion de la propriété intellectuelle, pressée en cela par les agences gouvernementales qui la considèrent comme un acteur socio-économique central dans la nouvelle économie du savoir. Signe de la grande actualité et de l'importance de l'enjeu, l'Organisation de coopération et de développement économiques (OCDE) publiait en 2003 une enquête sur les meilleures pratiques à l'échelle internationale en matière de propriété intellectuelle dans les organisations publiques de recherche. Les déclarations d'invention, les accords de licence et la création d'entreprises dérivées (spin-off industriel et spin-out ou spin-off universitaire) s'avéraient être prioritaires pour cette organisation ainsi que pour de nombreux gouvernements.

Même si l'idée de valorisation de la recherche dans le contexte d'une économie dite du savoir semble souvent aller de soi pour le développement économique des régions voire même pour la compétitivité au niveau mondial, elle implique d'importants changements institutionnels. Réduire l'idée de valorisation à la commercialisation de la recherche n'est pour nous qu'une des voies possibles de transformation de l'université. La question de la place et du rôle des universités dans la société est actuellement un enjeu de débat pour les acteurs politiques, universitaires et industriels qui mesurent l'implication des nouvelles pressions socio-économiques qui pèsent aujourd'hui sur les institutions (Conceiçao \& Heitor, 2001, p. 32; Etzkowitz \& Leydesdorff, 2000, p. 109). À cet égard, certains auteurs, tels Etzkowitz et al. (1998), parlent d'une nouvelle mission pour l'université. Comment celle-ci s'inscrit-elle à travers les changements de contexte et s'accommode-t-elle des missions traditionnelles d'enseignement et de recherche des universités? Empruntant une perspective à la fois descriptive 
et analytique, c'est à cette question que nous tenterons de répondre en portant une attention particulière aux contextes canadien et québécois.

Notre propos est que la question de la valorisation, souvent restreinte à celle de la commercialisation, est mal posée lorsqu'elle l'est en termes de nouvelle mission pour l'université. Les pratiques d'enseignement et de recherche, au cœur des missions traditionnelles de l'institution universitaire, se transforment dans le nouveau contexte de l'économie du savoir et du phénomène de la globalisation (Breton, 2003; Gibbons, 2003) et ne sont pas complètement distinctes des pratiques de valorisation. Il convient donc de bien définir la valorisation et le mandat même de l'université dans le contexte actuel. Au regard des débats que ces transformations suscitent, nous considérons qu'il s'avère fort pertinent de se questionner sur l'effet d'une implication accrue de l'université elle-même dans la définition de ce qu'elle sera demain, de la place qu'elle devra occuper et du rôle qu'elle devra jouer, à l'heure où il semble que les transformations qu'elle connaît lui sont largement imposées de l'extérieur.

Dans une première partie, nous préciserons les enjeux du développement de la valorisation de la recherche universitaire pour l'économie et la société, puis dans une deuxième partie nous présenterons les grandes lignes des politiques canadiennes et québécoises de développement de la valorisation de la recherche. Enfın, dans une dernière partie nous défendrons l'idée que le développement des activités de valorisation dépend notamment de la capacité des universités à s'impliquer, au travers de réflexions stratégiques internes, dans la définition de leurs rôles dans une économie du savoir.

\section{Le contexte économique de la valorisation de la recherche}

Lorsque la question des missions de l'université est abordée, il importe de prendre la mesure du contexte dans lequel elle évolue. Les missions de l'université ne sont pas statiques, les pratiques qui les incarnent changent dans le temps pour s'adapter à l'évolution de la société. En outre, l'idée de valorisation de la recherche universitaire demeure un phénomène récent. Au lendemain de la Deuxième Guerre mondiale, la représentation courante est plutôt que les missions de l'université par rapport à celles des institutions politiques, économiques et culturelles, peuvent être cernées de façon claire. L'université est vue dans cette période comme étant largement préservée des pressions environnementales. Elle semble autonome par rapport au contexte socio-économique, hors du jeu concurrentiel. Les savoirs qu'elle produit sont considérés comme étant un des éléments expliquant le développement de l'industrie et des marchés. La diffusion de ces savoirs dans la sphère socio-économique se fait par le biais de la publication et de la formation. Le gouvernement n'aurait longtemps pour rôle, à cet égard, que de réguler les échanges et de financer l'université sans conditions afin qu'elle poursuive sa mission sociale (OCDE, 1999).

L'université d'aujourd'hui, dans le contexte de la globalisation, de l'économie du savoir et des nouveaux modes de production des connaissances, ne peut plus se penser comme fonctionnant en vase clos. Elle doit s'inscrire dans des réseaux 
plus vastes pour mener à bien ses missions de recherche et d'enseignement (Breton, 2003). Les activités de recherche sont également plus complexes. Si la notion de territoire géographique est difficile à tenir dans une économie globalisée, il en va de même des territoires disciplinaires qui organisent la recherche et l'enseignement à l'intérieur de l'université (Gibbons, 2003, p.119). L'université opère aujourd'hui dans un environnement transdisciplinaire dans lequel les unités académiques ne peuvent plus, seules, faire le tour d'un champs intellectuel. Dans les réseaux d'échange de connaissances qui se développent, l'université n'est plus la seule organisation qui produit et diffuse des connaissances. Finalement, dans une économie du savoir, c'est le statut de la connaissance qui change lorsque cette dernière peut être considérée aussi comme une marchandise qui s'échange, se vend et s'achète (Breton, 2003, p. 28).

\section{Une évolution globale des modes de production des connaissances}

Les analyses des transformations des systèmes scientifiques et techniques dans les économies contemporaines se fondent sur des constats empiriques tels que l'accélération du rythme de transition vers une économie du savoir, les liens de plus en plus étroits entre la science et la technologie, le resserrement des relations entre les entreprises et les laboratoires publics, le développement de la concurrence internationale, le développement des stratégies de propriété intellectuelle, les restrictions budgétaires auxquelles sont confrontés les gouvernements, l'impact de ces restrictions sur les schémas de financement de la recherche universitaire et la hausse des coûts de la recherche en général (Slaughter \& Leslie, 1997).

Dans tous les pays de l'OCDE, l'université fait l'objet de nouvelles politiques gouvernementales qui lient la recherche, ses activités et ses produits à l'activité économique. De fortes pressions se font sentir afın que les universités entreprennent des activités de valorisation des résultats de leurs recherches.

Selon Gibbons et al. (1994), l'université est amenée à se positionner face à l'importance croissante de "nouveaux concurrents" dans la production de connaissances scientifique. L'université, où la recherche est organisée sur le mode disciplinaire et évaluée par la communauté restreinte des pairs, perd le monopole de la production scientifique au profit de nombreux autres sites de recherche (centres de recherche, laboratoires industriels, firmes de consultants, etc.). Cette réalité correspondrait au " mode 2 ». Alors que le mode 1 renverrait à un financement essentiellement institutionnel, à des intérêts académiques en contexte disciplinaire où les lieux de production seraient homogènes et où les chercheurs, évalués par leurs pairs, bénéficieraient d'une autonomie relative, le mode 2 renverrait à des modes de financement provenant d'une variété de sources publiques et privées mobilisées autour d'un projet, à des champs de recherche transdisciplinaires et hétérogènes dont le contrôle de la qualité se ferait par un ensemble d'acteurs ayant des intérêts intellectuels, économiques et/ou politiques dans la production de connaissances. L'évaluation de l'impact sociétal des recherches se ferait ici ex ante, c'est-à-dire lors de la définition des 
problèmes à résoudre et de l'établissement des priorités de recherche. Ainsi, le paradigme que l'on associe au mode 1 serait remis en cause en raison de l'impératif des contextes d'application locaux et spécifiques, soit en fonction des attentes et des valeurs des utilisateurs.

Gibbons et al. (1994) n'ont pas appuyé empiriquement leur thèse. Malissard (2000) montre que dans certains contextes le mode 2 apparaît bien avant l'après-guerre, et nombreuses sont les critiques qui concluent à la cohabitation et à la confrontation des deux modes (Dalpé \& Ippersiel, 2000; Shinn, 2000). Cependant, la conceptualisation de la production scientifıque qui est légitimée depuis quelques années par les pouvoirs publics intègre bien les "pressions " contextuelles qui s'exercent sur les universités. Sous l'influence notamment de la thèse normative de Gibbons et al. (1994), il semble y avoir un mouvement convergent dans la transformation des universités.

Etzkowitz, Webster, Gebhardt et Cantisano Terra (2000), suite à une analyse historique et comparative, concluent que les transformations dans plusieurs pays semblent converger vers un modèle d'université entrepreneuriale (Clark, 1998), malgré les résistances, les critiques, les problèmes institutionnels et les questions de gouvernance que cette évolution pose. Dans les pays de l'OCDE, les nouvelles politiques gouvernementales apparaissent étroitement liées à une reconfiguration majeure de la place et du rôle des universités dans la mondialisation de la production, de la diffusion et de l'utilisation des connaissances.

Le rapport [de l'OCDE, 1999] insiste sur la nécessité pour les universités d'agir avec plus de flexibilité afın de pouvoir « prospecter les marchés de la recherche " et " monnayer leurs compétences " de manière à ce que l'entreprenariat universitaire, avec ses équipes de chercheurs-entrepreneurs, soit en mesure d'assurer sa compétitivité avec des firmes mondiales "faisant leur marché " en matière de recherche là où les compétences se trouvent au meilleur rapport qualité/prix. (Milot, 2003, p. 72)

Dans cette perspective, c'est non seulement la recherche qui est envisagée de manière pragmatique mais également ses institutions, faisant en sorte que l'université a pour fonction de soutenir l'économie nationale, de produire des connaissances pour les industries du savoir et de fournir du personnel qualifié aux entreprises.

Le mouvement de valorisation semble prendre une ampleur sans précédent et être étroitement associé à l'idée de commercialisation, parfois de marchandisation de la recherche.

\section{Clarification du concept de valorisation}

Il convient de clarifier la notion de valorisation de la recherche, avant de nous attacher à l'analyse de son impact sur les missions de l'université. Selon le Conseil de la science et de la technologie (CST) du Québec (2005), qu'elle relève 
d'objectifs sociaux ou économiques, la valorisation " semble faire partie de ces expressions employées couramment, mais dont la difficulté à en préciser le sens apparaît aussitôt qu'on s'attarde à vouloir le cerner " (p.2). Le terme de valorisation se trouve souvent utilisé de façon interchangeable avec ceux de commercialisation (des résultats de la recherche universitaire, de la propriété intellectuelle ou technologique) et de transfert (technologique ou de connaissances). Ces termes renvoient pourtant à des réalités différentes. Le CST (2005) propose une clarification en reprenant les défınitions données par des organismes nationaux d'Amérique du Nord, d'Europe et d'Australie reconnus comme experts dans le domaine. Il donne dans un premier temps une définition générale de la valorisation.

$\mathrm{Au}$ sens premier du terme, la valorisation désigne le fait de donner une valeur ajoutée aux activités usuelles de la recherche et à ses résultats. Valoriser la recherche, c'est lui conférer une valeur autre que celle qu'elle a déjà, c'est rendre opérationnels (valeur d'usage) ou commercialisables (valeur d'échange) les connaissances, les compétences et les résultats de recherche. (CST, 2005, p. 9)

La valorisation apparait ainsi comme un terme générique qui comprend ceux de commercialisation et de transfert.

En fait, la valorisation peut être divisée en deux grands champs : d'une part, celui de la valorisation financière, commerciale ou économique et, d'autre part, celui de la valorisation sociale de la recherche (ou à visée non marchande). (CST, 2005, p. 9)

Ainsi, lorsque la question de la valorisation est abordée dans une perspective comparative, il convient de préciser le sens que prend le concept de valorisation selon les pays. Dans la plupart des pays de l'OCDE, il est associé à l'idée de commercialisation des résultats de la recherche universitaire alors qu'au Québec, par exemple, le concept de valorisation prend un sens tout autant social que technologique. La politique dont s'est dotée cette province canadienne concerne autant les sciences humaines et sociales que les sciences naturelles et le génie (Milot, 2005, p. 26).

\section{Des discours normatifs à l'échelle internationale}

Les pressions politiques visant à inscrire davantage l'université dans l'environnement concurrentiel sont très fortes. À cet effet, le rapport de $2003 \mathrm{de}$ l'OCDE sur les " Technology Transfer Offices " donnait lieu à quatre recommandations : rendre les politiques nationales en matière de propriété intellectuelle plus cohérentes, encourager le développement et l'implantation de politiques en matière de propriété intellectuelle au niveau des institutions, augmenter les capacités en gestion de la propriété intellectuelle des organismes publics de recherche, et améliorer la collecte des données et partager les pratiques exemplaires. 
Les politiques technologiques convergent vers la promotion des activités menées en coopération entre les secteurs public et privé. La collaboration devient ce faisant un outil de gestion dans les programmes gouvernementaux d'innovation. À titre d'exemple, les universités canadiennes collaboraient déjà activement à la fin des années 90 avec l'industrie. Celle-ci sous-traitait $5 \%$ de son travail de recherche et développement (RD) aux universités et finançait $12 \%$ des autres travaux de RD réalisés dans les universités (Godin \& Gingras, 1999). Les pratiques de partenariat et d'entrepreneurship permettent aux universités de s'ajuster aux changements et aux gouvernements d'atteindre leurs nouveaux objectifs. Les différents gouvernements cherchent à développer une culture entrepreneuriale au sein des universités, culture qui doit se traduire par l'augmentation du nombre d'entreprises dérivées, de centres de recherche interdisciplinaires et de parcs scientifiques (Conceiçao \& Heitor, 1998).

La commercialisation de la recherche tend ce faisant à s'imposer comme une nouvelle mission de l'université. Selon Scott (2003, p. 234), si les universités ne s'adaptent pas à ce nouveau contexte, elles deviendront rapidement des " anachronismes ", voire même des " épaves institutionnelles ". Pourtant, les universités ont déjà transformé leur manière de faire de la recherche et d'en diffuser les résultats. Une bonne partie des pressions visant l'adoption d'une nouvelle mission de valorisation provient des universités elles-mêmes (Gingras, 2003, p. 6), si bien qu'il est délicat d'envisager que cette nouvelle mission soit simplement plaquée sur de traditionnelles missions d'enseignement et de recherche qui n'auraient pas changé depuis le début du dix-neuvième siècle. C'est pourtant souvent de cette façon que les débats portant sur les nouveaux rôles de l'université sont engagés, faisant oublier que la valorisation peut aussi prendre de multiples formes et se pratique non seulement par le biais de la recherche mais également par le biais de l'enseignement. Ainsi, le développement des pratiques de valorisation passe également par une évolution des modes d'évaluation des activités de l'université, afın de favoriser le développement de la recherche effectuée en réseaux, de participer à la gestion de cette recherche en s'assurant de sa pertinence et du respect du bien commun et de continuer à former une élite intellectuelle.

\section{Les politiques canadiennes et québécoises de valorisation de la recherche}

Afın de préparer la société canadienne à affronter les défis économiques et sociaux de ce siècle, le gouvernement fédéral canadien s'est engagé ces dernières années dans une politique de soutien à la recherche, à l'innovation et au développement des compétences (Association des universités et collèges du Canada, 2001). Le Québec s'est doté en 2001 d'une politique de la science et de l'innovation (Ministère de la Recherche, de la Science et de la Technologie, 2001) et d'un Plan d'action en matière de gestion de la propriété intellectuelle en 2003 (MRST, 2002). Il a notablement développé ses activités de valorisation de la recherche. 
La politique canadienne de soutien à la recherche et à l'innovation

L'OCDE (2002) évalue à 126 milliards de dollars américains l'investissement international dans la RD universitaire. La recherche universitaire canadienne représente $5 \%$ de cet investissement total alors que le Canada ne représente que $0,6 \%$ du produit intérieur brut (PIB) mondial. Cet investissement massif en recherche a bénéficié en priorité aux secteurs où les possibilités de croissance sont les plus élevées: l'aéronautique, les produits pharmaceutiques, les biotechnologies, les technologies de l'environnement, les technologies des télécommunications et de l'information, les nanotechnologies et nanosciences et les technologies de fabrication de pointe (Pinot \& Marini, 2006). Les universités, qui produisent la majeure partie de la recherche publique, réalisent actuellement près de $30 \%$ de la RD du pays (ibid.).

Les investissements importants du gouvernement du Canada se sont également traduits par la création des Réseaux de centres d'excellence en 1989, de la Fondation canadienne pour l'innovation en 1999, du Programme de subvention des coûts indirects de la recherche en 2001, du Programme des chaires de recherche du Canada en 2000 et de la Politique canadienne de l'innovation en 2003. On assiste aussi à l'augmentation du financement des trois organismes subventionnaires : le Conseil de recherches en sciences naturelles et en génie du Canada, le Conseil de recherches en sciences humaines du Canada et les Instituts de recherche en santé du Canada.

Les structures en charge de la valorisation dépendent du ministère de l'Industrie du Canada (Industrie Canada) et du Conseil national de recherche du Canada. Les mesures prises en termes de valorisation se traduisent par des investissements dans l'infrastructure du transfert de technologie, la divulgation et la commercialisation de la propriété intellectuelle, la diffusion des bonnes pratiques de la valorisation ainsi que la diffusion d'indicateurs de développement de la valorisation de la recherche universitaire. La gestion de la valorisation relève de différentes stratégies. À cet effet, on retrouve des stratégies de gestion de la propriété intellectuelle par la voie des bureaux de transfert de la technologie appartenant aux universités, d'organismes distincts sans buts lucratifs ou de sociétés à but lucratif composées d'actionnaires et d'un conseil d'administration indépendant (Pinot \& Marini, 2006).

Selon l'Association des universités et collèges du Canada, les universités canadiennes s'engageaient à la fin des années 90 à tripler le rendement au plan de la valorisation pour les dix prochaines années et, ce, à condition que les gouvernements et l'industrie leur accordent un soutien adéquat. Au cours de la même période, le gouvernement fédéral s'engageait à ce que le Canada parvienne à être d'ici 2010 parmi les cinq meilleurs pays au monde en matière de RD en fonction de son PIB. En 2002, le Canada occupait le quinzième rang mondial.

L'Association of University Technology Managers (AUTM) sonde depuis 1991 ses établissements membres. Le rapport produit en 2006 (Stevens, Toneguzzo \& Bostrom, 2006) s'avère riche d'enseignements en ce qui concerne les dépenses 
en recherche, les inventions, les redevances et la création d'entreprises par les universités canadiennes membres de l'AUTM. Bien que le nombre de répondants ne soit pas le même d'une année à l'autre, de grandes tendances apparaissent au travers des enquêtes présentées.

Ainsi, pour les établissements universitaires membres de l'AUTM, les dépenses en recherche, financées par le gouvernement fédéral, l'industrie, les gouvernements provinciaux et locaux, les fondations, les individus et les institutions, ont progressé constamment de 1991 à 2004, passant de 580 millions de dollars canadiens à 4070 millions, avec une accélération de la croissance des dépenses à partir de 1999 (Stevens et al., 2006). Durant cette même période, le financement provenant du gouvernement fédéral a augmenté de 374 millions tandis que celui de l'industrie a augmenté de 42 millions.

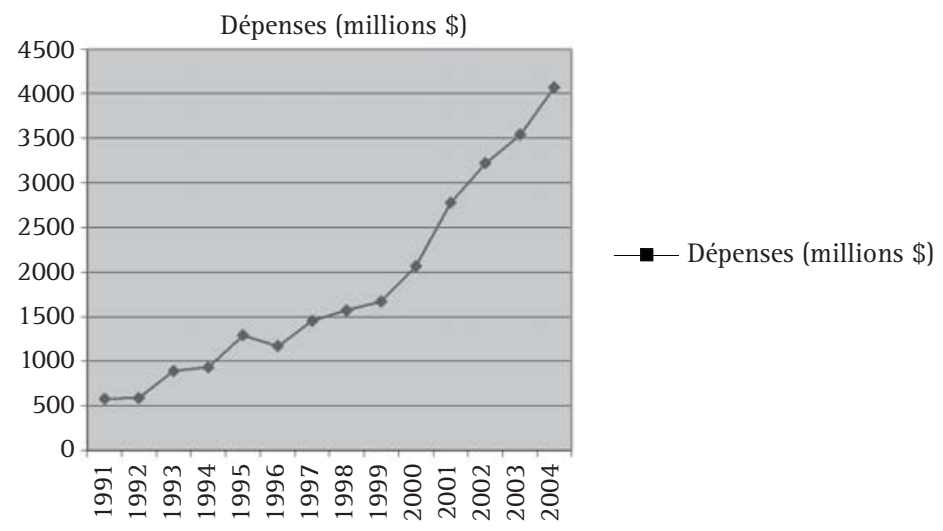

Figure 1 : Les dépenses en recherche des universités canadiennes membres de l'AUTM (d'après Stevens et al., 2006) ; dollars canadiens.

La part du gouvernement fédéral dans le financement total de la recherche des universités membres de l'AUTM est restée relativement stable depuis le milieu des années 90 et représente 48,3\% du financement total de la recherche pour l'année 2004. La part de l'industrie est quant à elle en baisse depuis 1997 et représente 11,3\% en 2004 (Stevens et al., 2006). Cette baisse s'explique par la forte augmentation des financements gouvernementaux et non par une diminution des ressources provenant de l'industrie. À titre de comparaison, notons qu'aux États-Unis le financement de la recherche pour 164 institutions membres de l'AUTM est évalué en 2004 à 44,4 milliards de dollars américains, dont les deux tiers proviennent du gouvernement fédéral et environ $8 \%$ de l'industrie.

Une enquête de Statistiques Canada (2003) montre que l'augmentation globale des financements de la recherche canadienne s'est effectivement accompagnée d'un développement de l'implication des universités dans la valorisation de la recherche. Parmi ces dernières, 62 \% sont dotées d'un bureau de commercialisation (bureau de développement des affaires, bureau de liaison 
entreprises-université ou bureau de transfert technologique) ou ont nommé des gestionnaires afın de gérer leur propriété intellectuelle.

Selon l'AUTM, la divulgation d'inventions au Canada présente une progression au cours de la période avec 250 divulgations pour 9 répondants en 1991 et 1307 divulgations pour 33 répondants en 2004. Par ailleurs, depuis 1980, 712 entreprises ont été créées par les universités participant à l'enquête. Toutefois, le nombre de créations annuelles présente une tendance à la baisse depuis 2001 .

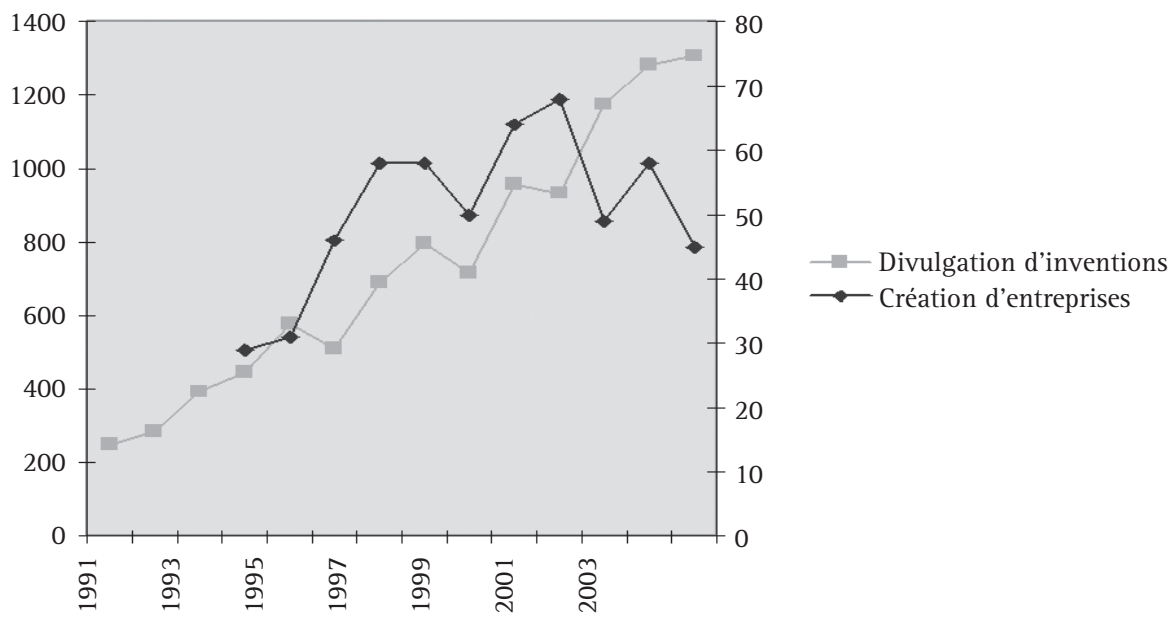

Figure 2 : Divulgations d'inventions et création d'entreprises des universités canadiennes (d'après Stevens et al., 2006)

Les redevances perçues pour l'année 2004 pour 33 répondants sont évaluées à 54,23 millions de dollars canadiens. L'augmentation constante de ces redevances depuis 1991 a nettement accéléré à partir de 1999. Cependant, elles ne représentent que $1,1 \%$ des fonds de recherche des universités canadiennes à la fin des années 90 et 1,3\% (4,07 milliards de dollars canadiens) pour 2004, ce qui constitue en somme une hausse de faible importance par rapport au financement global de la recherche.

Ces statistiques reflètent un certain virage entrepreneurial des universités canadiennes depuis le début des années 90 . Elles montrent également que si les revenus de la propriété intellectuelle des universités ont connu une augmentation importante depuis 1999, ils demeurent tout de même relativement faibles. Ainsi, malgré la progression et l'importance des investissements du gouvernement fédéral dans la recherche, la valorisation n'est pas une activité des plus lucratives, même si on observe une légère hausse du rendement depuis la fin des années 90. Bien que l'investissement en recherche ne puisse donner lieu sans délai à des retombées financières, il apparait clair que la pertinence de l'investissement dans la recherche universitaire ne peut être appréciée sur la seule base de sa rentabilité financière. Plus globalement, la vocation de cet investissement est de créer un climat favorable à l'innovation grâce aux multiples 
facettes de la contribution des universités à la création et à la diffusion de connaissances. La politique québécoise de valorisation de la recherche est abordée dans cette perspective.

Redevances (millions)

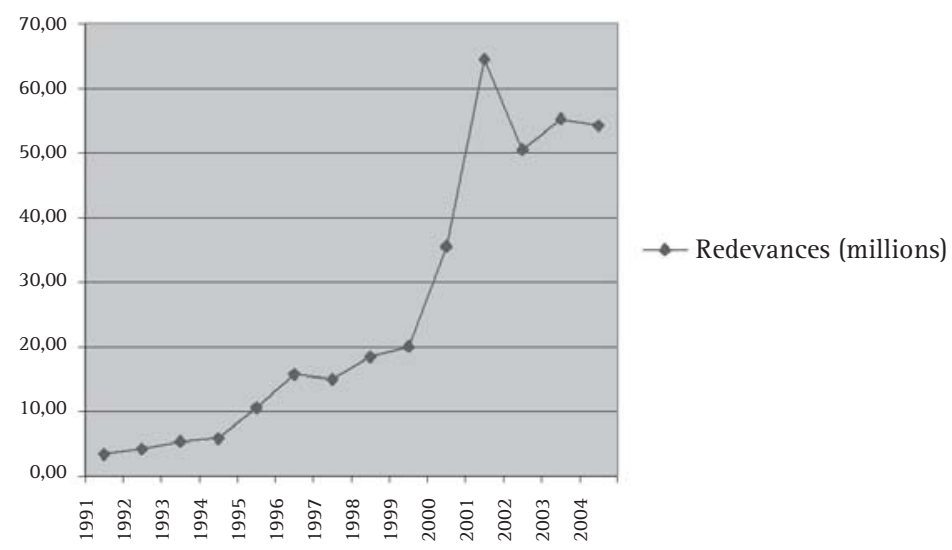

Figure 3 : Les redevances perçues par les universités canadiennes (d'après Stevens et al., 2006) ; dollars canadiens.

\section{La stratégie de valorisation québécoise}

Une caractéristique marquante des politiques d'innovation québécoises est la portée donnée à la valorisation de la recherche. Elle est comprise comme un ensemble d'activités destinées à favoriser l'innovation sur le plan social aussi bien que sur le plan technologique. La valorisation de la recherche ne concerne donc pas uniquement les connaissances scientifiques exploitables commercialement. Elle prend en considération l'ensemble des résultats de la recherche dans tous les domaines y compris les sciences sociales et humaines.

Dans le système québécois, la valorisation de la recherche universitaire se situe au sein de quatre missions : l'enseignement, la recherche, le service aux collectivités et l'innovation couvrant les champs technologique, social et culturel. La valorisation en tant que mode de mise en relation de la recherche et du milieu socio-économique renvoie en ce contexte à différents services, dispositifs et mécanismes internes et externes aux universités (CST, 2005). Au plan interne, les universités peuvent avoir des bureaux de liaison entreprise-université (les BLEU, au nombre de 18), des chaires de recherches institutionnelles et industrielles, des centres d'entrepreneurship universitaire (CEU, au nombre de 8) et bénéficier de différents programmes ciblés de partenariat. Au plan externe, on retrouve les sociétés de commercialisation ou de valorisation de la recherche universitaire au Québec (SVU, au nombre de 4), les centres de liaison et de transfert (CLT, au nombre de 7), les consortiums de recherche en partenariat et les parcs scientifiques et technologiques affiliés aux universités. 
L'organisme Valorisation-Recherche Québec a joué un rôle important dans le développement de la valorisation de la recherche. Créé pour la période 19992006 par le ministère de la Recherche, de la Science et de la Technologie avec un budget de 220 millions de dollars canadiens, il s'est donné pour objectif de renforcer la recherche et d'en accroître les retombées pour la société québécoise, en favorisant différents modes de contribution : un programme d'aide au démarrage d'infrastructures, une aide financière liée aux besoins d'infrastructure des chaires de recherche du Canada et des projets d'envergure jugés prioritaires pour la recherche au Québec, ainsi qu'un soutien aux projets d'envergure internationale favorisant l'interaction entre les chercheurs, les partenaires et les utilisateurs de connaissances. Trois formes de valorisation ont été envisagées : la valorisation commerciale avec les sociétés de valorisation, la collaboration multidisciplinaire avec le programme Soutien aux projets structurants et le transfert vers l'usager avec le programme Projets d'envergure. Quatre sociétés de valorisation regroupant des universités et des centres hospitaliers universitaires ont été créées avec l'appui de différents investisseurs: SOVAR (Université Laval et Centre hospitalier universitaire de Québec), VALEO (Concordia, École de technologie supérieure, Université du Québec à Montréal et Université du Québec à Rimouski), UNIVALOR (Université de Montréal, École Polytechnique, HEC Montréal et centres hospitaliers affilliés) et MSBi (Université McGill, Université de Sherbrooke, Université Bishop's et centres hospitaliers affiliés).

Un trait distinctif du système québécois tient aux réseaux de collaboration qu'il a réussi à mettre sur pied entre les différents centres de liaison et de transfert. Une des priorités de ce système est de favoriser l'insertion des universités dans des réseaux d'échanges de connaissances.

\section{Les limites des discours normatifs}

La question de la valorisation nécessite une mise en perspective des pressions et des discours normatifs qui tendent parfois à orienter les pratiques de recherche et d'enseignement à l'université vers la seule idée de commercialisation. La logique des pratiques exemplaires conduit à l'adoption d'indicateurs de mesure de la valorisation trop homogènes, inadaptés face à la diversité des contextes, des méthodes (Agrawal, 2001; Pries \& Guild, 2004), des ressources valorisables, des choix de structures et de gestion des universités (Milot, 2005; CST, 2005). Allant ainsi dans le sens de Gemme, Gingras et Godin (1999), nous proposons d'envisager le développement de la valorisation de la recherche en considérant que le véritable enjeu ne se réduit pas à des bénéfices financiers mais au rôle donné aux universités et à la place qu'elles doivent prendre dans la réorganisation des rapports sociaux qui structurent l'économie dite du savoir, dans des contextes nationaux et régionaux précis. 
Une perception étroite de la valorisation de la recherche

Gibbons, dans un discours sur l'enseignement supérieur du vingt-et-unième siècle destiné à la Banque Mondiale, affirme que les impératifs économiques sont aujourd'hui la référence contextuelle absolue et que : "Si les universités ne s'adaptent pas, on se passera d'elles " (Milot, 2003, p. 70). Certaines évolutions laissent penser que cette adaptation est en marche. Les universités ont ainsi multiplié ces dernières années leurs activités de recherche appliquée et se sont donné la recherche commercialisable comme préoccupation, dans le souci notamment de diversifier les sources de financement de la recherche (Faulkner \& Senker, 1995). Elles ont adopté trois ensembles de stratégies complémentaires en multipliant les accords de valorisation industrielle qui associent chercheurs publics et privés, en se donnant des politiques actives en matière de dépôt de brevet et de concession de licences et en développant rapidement des essaimages académiques (Hamdouch \& Depret, 2001, p. 146). Certains groupes de recherche universitaires en sont même venus à être comparés à des "quasi-firmes " au sein desquelles le directeur consacre la majeure partie de son temps à diriger des post-doctorants et chercher des fonds plutôt que de faire de la recherche (Gingras, 2003). De ce point de vue, l'université entrepreneuriale émerge en partie sur des bases internes à l'université. Malissard, Gingras et Gemme (2003) récusent l'argument selon lequel les universités ne font que s'adapter aux nouvelles réglementations de manière passive en montrant qu'elles n'ont pas attendu ces politiques pour se transformer et se montrer plus entreprenantes au niveau économique.

David, Mowery et Steinmueller (1994) critiquent vivement ces tentatives de faire jouer un rôle aux universités dans la valorisation de connaissances. Ils questionnent cette très forte volonté des gouvernements de faire des universités des instruments des politiques nationales de R\&D, des joueurs dans les stratégies de rivalité, puisque cela n'est pas sans conséquence sur leurs missions traditionnelles. La question posée est celle de la possibilité de rendre compatibles les objectifs de la recherche fondamentale et de la recherche commercialisable. Ainsi, Caraça (2002) s'interroge sur la capacité de l'institution universitaire à se métamorphoser à nouveau, après avoir ajouté la mission de la recherche à celle de l'enseignement au dix-neuvième siècle.

Les risques liés aux discours normatifs actuels sont de n'aborder la valorisation de la recherche qu'à travers sa dimension financière, dans une logique fondée sur une perception appauvrie du rôle des universités dans la société. Cette logique conduit à opposer catégoriquement la valorisation de la recherche aux missions traditionnelles de l'université. Cette opposition conduit les uns à vouloir que l'université se structure comme une entreprise poursuivant un objectif ultime de rentabilité et conduit les autres à rejeter en bloc la valorisation de la recherche en raison des incompatibilités que le financement privé de la recherche présente avec la libre diffusion des connaissances dans la société.

En replaçant la question de la valorisation dans le contexte d'une évolution des modes de production et de diffusion des connaissances et en distinguant 
l'idée de valorisation et celle de commercialisation comme le suggère le Conseil de la science et de la technologie du Québec, nous pouvons aborder le débat du développement de la valorisation de la recherche en posant la question de l'insertion des universités dans les nouveaux réseaux de production et de diffusion du savoir. Ainsi envisagé, le rôle de l'université apparaît alors beaucoup plus riche que celui de générer des bénéfices grâce à la création et à la diffusion de connaissances.

Comme le souligne Gibbons (2003, p. 121) la recherche est une activité largement pratiquée, avec une compétence reconnue, hors des murs de l'université aujourd'hui. Cependant, ce sont les universités qui, toujours, forment les chercheurs qualifiés grâce aux compétences et à l'expertise présentes dans les réseaux de recherche. La question de la valorisation à l'université semble ainsi indissociable de celle de la formation universitaire. Pour van Kingel (2003), l'enseignement supérieur joue un rôle fondamental dans l'économie aujourd'hui parce qu'il forme des enseignants, des médecins, des experts dans l'organisation des services de santé, du système juridique, de l'administration, de l'industrie. Seules les universités combinent, par le biais des programmes de deuxième et de troisième cycle, la recherche et la formation dans un cadre unique de formation à la recherche par la recherche. L'enseignement supérieur contribue au développement des capacités intellectuelles et des capacités d'apprentissage nécessaires dans les systèmes de production et d'utilisation du savoir, que ce soit à travers la formation à la recherche ou la formation des acteurs de l'économie de demain à des pratiques professionnelles dans tous les domaines.

Lundvall (2002) souligne un autre rôle fondamental que seules les universités peuvent continuer à assumer grâce à la part qu'elles jouent dans la production de connaissances. Elles sont le seul lieu où pourraient être préservées les capacités à développer des réflexions critiques, des apprentissages en profondeur, des perspectives à long terme, bref, un lieu de formation des élites intellectuelles capables de prospecter, de penser l'avenir de nos sociétés. Laisser les universités jouer ce rôle fondamental pour la société suppose que l'efficacité de la valorisation de la recherche ne soit pas seulement perçue sous un angle financier, mais également sous l'angle des retombées plus globales de la science sur la société, qui justifie la continuité du soutien public à la recherche. Ne pas réduire la valorisation de la recherche scientifique à sa dimension financière suppose également de ne pas considérer la commercialisation de la recherche comme une mission en soi, mais comme un moyen parmi d'autres dont dispose l'université pour accroître les retombées de ses activités de création de connaissances sur la société.

\section{Les pratiques exemplaires et la spécificité des universités}

Beaucoup de pratiques considérées comme exemplaires en valorisation de la recherche sont issues de l'expérience des universités américaines. L'impact du modèle américain dans les schèmes interprétatifs des universités à l'échelle internationale fait l'objet de débats (Milot, 2005) avec notamment la remise 
en cause de l'importance donnée à la loi Bayh-Dole dans le développement de l'entrepreneurship universitaire (Mowery, 1998). Si l'identification des bonnes pratiques nous ramène à la question précédemment évoquée de la définition des objectifs de l'université et de la place donnée à la valorisation de la recherche par rapport aux missions de l'université, elle pose également la question de la pertinence des comparaisons entre universités.

Bien que toutes les universités connaissent des contraintes similaires liées à l'évolution de la production des connaissances dans la société, chacune œuvre dans un environnement spécifique et présente des caractéristiques qui lui sont propres (Mailhot \& Schaeffer, 2005). Ainsi, elles ne peuvent peut-être pas toutes s'adapter de manière identique. Les disparités entre universités tiennent à une multitude de facteurs tels que le niveau de développement des régions, le rôle donné aux différentes organisations dans les systèmes d'innovation nationaux, les politiques publiques de propriété intellectuelle et de stimulation de création d'entreprises, le financement des agences gouvernementales, des conseils subventionnaires et des administrations locales, le degré de développement et de synergie des réseaux industriels, les ressources et le niveau d'investissement de capital de risque des universités, les compétences des bureaux de transfert internes ou externes aux universités, le niveau de concurrence entre les universités, les orientations des champs de recherche des universités, la diversité et la qualité des membres du corps professoral, le temps que ces derniers consacrent à la recherche, ainsi que le caractère cumulatif et tacite d'une part importante de leurs connaissances. Selon Milot (2005), il faut comparer ce qui est comparable lorsqu'il est question des "meilleures pratiques " à l'échelle internationale.

La question de la comparaison pose celle de la spécificité des stratégies que les groupes de recherche universitaires peuvent adopter face à l'évolution de leur environnement scientifique ou technologique. Chaque université dispose d'un ensemble d'actifs construit au travers des activités passées : d'une part, les activités de recherche qui ont conduit à l'accumulation de compétences spécifiques dans certaines disciplines et, d'autre part, les activités de valorisation qui ont permis de développer des ressources spécifiques liées à la connaissance de la société, des entreprises, des marchés et à la constitution de réseaux.

Par ailleurs, la valorisation de la recherche est multiforme et les différents niveaux d'intervention politique incitent les universités à prendre en charge des activités de valorisation. Ces activités répondent à des orientations multiples telles que la diversification des financements de la recherche académique, la diffusion de connaissances à travers les activités de formation, le soutien à l'innovation des entreprises locales ou l'exploitation directe des connaissances par la création d'entreprises. Selon les axes de valorisation développés, les modifications à apporter au mode de fonctionnement des universités sont plus ou moins profondes et donc susceptibles de remettre en cause la cohérence globale si elles ne sont pas gérées. Selon que l'université mette l'accent dans sa politique de valorisation sur une finalité scientifique (la création et la diffusion de connaissances scientifiques), éducative (la formation des étudiants et des cherch- 
eurs), financière (la réalisation d'un profit), économique (le développement du tissu industriel) ou de service (la participation des chercheurs à la création de réseaux de recherche), la nature des objectifs poursuivis est différente. Le fait d'inciter les universités à adopter une réflexion plus stratégique ouvre peut-être la porte à ce qu'elles prennent véritablement position dans les nouveaux rapports qui se dessinent entre le champ universitaire et économique.

Dans le "régime de la science stratégique " (Rip, 2002) où les pressions sont de plus en plus fortes pour que les universités intègrent une troisième mission de valorisation de la recherche à côté de leurs missions de recherche et d'enseignement, un modèle de gestion plus stratégique que collégial semble s'imposer aux universités, qui ne peuvent s'adapter uniformément face à ces pressions de portée globales.

\section{CONCLUSION}

Les questions liées au développement de la valorisation de la recherche gagneraient à être posées autrement qu'en termes de mission. Le fait de considérer la valorisation de la recherche comme une mission qui se juxtapose aux missions traditionnelles de l'université conduit à isoler l'ensemble des activités s'y rapportant dans une logique purement financière. Or, l'analyse des risques liés à une adoption globale des discours normatifs montre qu'il conviendrait de prendre la mesure des changements en matière de recherche et d'enseignement universitaires dans différents contextes. Les indicateurs utilisés pour rendre compte du développement de la valorisation de la recherche sont essentiellement économiques et ne permettent pas de faire avancer la réflexion. Des indicateurs tels que la part du financement industriel de la recherche, le nombre d'inventions divulguées, d'entreprises créées ou le montant des redevances réduisent la valorisation à la commercialisation de la recherche et ne font que traduire un niveau d'activité des universités. Ainsi que le souligne Godin (2004) il est nécessaire de mener une réflexion spécifique pour définir des indicateurs adaptés aux organisations innovantes de la sphère non marchande pour lesquelles l'innovation ne passe pas nécessairement uniquement par l'innovation technologique. Pour ce type d'organisation, dont les universités font partie, les indicateurs devraient d'avantage être orientés vers la mesure de l'impact des différentes activités de valorisation sur l'environnement propre à chacune. Dans ce débat, il semble aussi que les universités demeurent assez muettes, se positionnant surtout dans un rôle de spectateur plutôt que d'acteur (Breton et Lambert, 2003). Or, si les pressions actuelles obligent les universités à adopter des modes de gestion plus stratégiques, cela implique aussi une attitude plus volontariste à laquelle elles ne sont peut-être pas habituées.

Les discours politiques se veulent largement déterministes et très pragmatiques. Ce sont pourtant les universités qui peuvent le mieux évaluer la nature de leur contribution sociale. L'analyse de la valorisation de la recherche montre qu'elle implique une réorganisation des rapports sociaux, une transformation de l'institution universitaire et de son esprit. Malgré les précautions argumen- 
tatives que l'on retrouve dans les documents gouvernementaux concernant la différence entre la commercialisation et les activités de valorisation de la recherche, notre examen des mesures, outils et stratégies universitaires de valorisation a montré qu'il y a des risques d'évaluer le développement de la valorisation de la recherche sur la base d'indicateurs essentiellement focalisés sur la dimension financière de la valorisation de la recherche. L'université, plutôt que de demeurer un lieu de production de réflexions fondamentales, un lieu de recul et d'élaboration d'une pensée critique, deviendrait plutôt une organisation de production de connaissances utiles à l'industrie, donnant préséance à la recherche dans certains domaines tels que le génie et les sciences naturelles et à des recherches sujettes à une exploitation commerciale. Ainsi, la question à laquelle aboutit clairement le développement de la valorisation est celle de la raison d'être de chaque université dans son environnement propre.

\section{RÉFÉRENCES}

Agrawal, A.K. (2001). University-to-industry knowledge transfer: literature review and unanswered questions. International Journal of Management Reviews, 3, 285-302.

Association des universités et des collèges canadiens. (2001). La valorisation de la recherche universitaire. Document de réflexion. Consulté le 06 février 2007: http://www.aucc.ca/_pdf/français/reports/2001/commerc_05_25_f.pdf.

Breton, G., \& Lambert, M. (Eds.) (2003). Globalisation et universités. Nouvel espace, nouveaux acteurs. Éditions UNESCO, Les Presses de l'Université Laval.

Breton, G. (2003). De l'internationalisation à la globalisation de l'enseignement supérieur. Dans Globalisation et universités. Nouvel espace, nouveaux acteurs, (p. 21-34). Éditions UNESCO, Les Presses de l'Université Laval.

Caraça, J. (2002). Introductory note: should universities be concerned with teaching or with learning? In P. Conceiçao, D. Gibson, M. V. Heitor, G. Sirilli, \& F. Veloso (Eds.), Knowledge for Inclusive Development (p. 31-34). Westport and London : Quorum Books.

Clark, B. R. (1998). Creating entrepreneurial universities: organizational pathways of transformation. AIU and Elvesier.

Conceiçao, P., \& Heitor, M. V. (2001). Universities in the learning economy: balancing institutional integrity with organizational diversity. In D. Archibugi, \& B.-A. Lundvall (Eds.), The globalizing learning economy (pp. 49-78). Oxford : Oxford University Press.

Conceiçao, P., \& Heitor, M. V. (1998). On the role of the university in the knowledge economy. Science and Public Policy, 26, 37-51 
Conseil de la science et de la technologie du Québec, (2005, février). La valorisation de la recherche universitaire; clarification conceptuelle. Gouvernement du Québec.

Dalpé, R., \& Ippersiel, M.-P. (2000). Réseautage et relations avec l'industrie dans les nouveaux matériaux et l'optique. Sociologie et Sociétés, 32(1), 107134.

David, P. A., Mowery D. C., \& Steinmueller, W. E. (1994). University-industry research collaborations: managing missions in conflict. Conference on "University goals, institutional mechanisms, and the 'industrial transferability' of research", Center for Economic Policy Research, Stanford University, 18-20.

Etzkowitz, H., A., Webster, C. G., \& Cantisano Terra, B. R. (2000). The future of the university and the university of the future: evolution of ivory tower to entrepreneurial paradigm. Research Policy, 29, 313-330.

Etzkowitz, H., \& Leydesdorff, L. (2000). The dynamics of innovation : from national systems and "Mode 2" to a Triple Helix of university-industry-government relations. Research Policy, 29, 109-124.

Etzkowitz, H., Webster, A., \& Healey, P. (1998). Capitalizing knowledge. New intersections of industry and academia. State University of New York Press.

Faulkner, W., \& Senker, J. (1995). Knowledge frontiers. Oxford : Oxford University Press.

Gemme, B., Gingras, Y., \& Godin, B. (1999). La commercialisation de la recherche universitaire: que disent vraiment les chiffres? (Note de recherche du CIRST 1999-10). Université du Québec à Montréal.

Gibbons, M. (2003). L'avenir de l'enseignement supérieur dans un monde globalisé. Dans Globalisation et universités. Nouvel espace, nouveaux acteurs (p.117-128). Éditions UNESCO, Les Presses de l’Université Laval.

Gibbons, M., Limoges, C., Nowotny, H., Schwartzman, S., Scott, P., \& Trow, M. (1994). The new production of knowledge. The dynamics of science and research in contemporary societies. London : Sage Publications.

Gingras, Y. (2003). Idées d'universités. Enseignement, recherche et innovation. Actes de la recherche en sciences sociales, 148, 3-7.

Godin, B. (2004). L'organisation innovante : d'un système d'indicateurs appropriés. Montréal : Consortium canadien sur les indicateurs de science et d'innovation.

Godin, B., \& Gingras, Y. (1999, juillet). L'impact de la recherche en partenariat sur la production scientifique. Dossier de Recherche, 3, 3. Ottawa : Association des universités et collèges du Canada.

Hamdouch, A., \& Depret, M. H. (2001). La nouvelle économie industrielle de la pharmacie. BioCampus, Editions Scientifiques et Médical Elsevier. 
Lundvall, B.-A. (2002). The university in the learning economy (DRUID Working Papers, No. 6). Copenhagen Business School.

Mailhot, C., \& Schaeffer, V. (2005). Universities specificities and the emergence of a global model of university: how to manage these contradictory realities. In P. Llerena, \& M. Matt (Eds), Innovation policy in a knowledge-based economy (p. 339-359). Berlin : Springer.

Malissard, P. (2000). Les "Start-up " de jadis: la production de vaccins au Canada. Sociologie et Sociétés, 32(1), 93-106.

Malissard, P., Gingras, Y., \& Gemme, B. (2003). La commercialisation de la recherche. Actes de la recherche en sciences sociales, 148, 57-67.

Milot, P. (2003). La reconfiguration des universités selon l'OCDE. Économie du savoir et politique de l'innovation. Actes de la Recherche en Sciences Sociales, 148, 68-73.

Milot, P. (2005). La commercialisation des résultats de la recherche universitaire : une revue de littérature (CIRST, no. 2005-01). Montréal : Centre interuniversitaire de recherche sur la science et la technologie.

Ministère de la recherche, de la Science et de la Technologie. (2001). Politique québécoise de la science et de l'innovation. Savoir changer le monde. Gouvernement du Québec.

Ministère de la Recherche, de la Science et de la Technologie. (2002). Plan d'action. Gestion de la propriété intellectuelle dans les universités et les établissements du réseau de la santé et des services sociaux où se déroulent des activités de recherche. Gouvernement du Québec.

Mowery, D. (1998). The changing structure of the US national innovation system : implications for international conflict and cooperation in R\&D policy. Research Policy, 27, 639-654.

OCDE. (1999). The management of science systems. Paris : OCDE/STI.

OCDE. (2002). Principaux indicateurs de la science et de la technologie, 2. Paris : OCDE.

OCDE. (2003). Turning science into business. Patenting and licensing at public research organizations. Paris : OCDE.

Pinot, E., \& Marini, J.-F. (2006, juillet). Financement de la recherche et $d u$ secteur science et technologie au Canada. Ambassade de France au Canada : Mission pour la Science et la Technologie.

Pries, F., \& Guild, P.D. (2004). Analyzing the commercialization of university research: A proposed categorization scheme. Washington, DC : 13th Annual Meeting of the International Association for the Management of Technology.

Rip, A. (2002). Regional Innovation Systems and the Advent of Strategic Science. Journal of Technology Transfer, 27,123-131. 
Scott, P. (2003). Acteurs en mutation dans une société du savoir. Dans G. Breton, \& M. Lambert, (Eds.), Globalisation et universités. Nouvel espace, nouveaux acteurs (p.233-246). Éditions UNESCO, Les Presses de l'Université Laval.

Shinn, T. (2000). Axes thématiques et marches de diffusion. La science en France, 1975-1999. Sociologie et Sociétés, 32 (1), 41-69.

Statistiques Canada. (2003). Enquête sur la commercialisation de la propriété intellectuelle dans le secteur de l'enseignement supérieur, 2001. Ottawa : Gouvernement du Canada.

Stevens, A. J., Toneguzzo, F., \& Bostrom D. (Eds). (2006). AUTM Canadian licensing survey, FY 2004 survey summary. Association of University Technology Managers (AUTM).

Van Ginkel, H. (2003). Que signifie la globalisation pour les universités? Dans Globalisation et universités. Nouvel espace, nouveaux acteurs (p. 75-86). Éditions UNESCO, Les Presses de l'Université Laval.

\section{COORDONNÉES}

Chantale Mailhot, Ph.D.

HEC - Montréal

Service de l'enseignement du management

3000 Chemin de la Côte-Sainte-Catherine

Montréal (Québec) Canada H3T 2A7

chantale.mailhot@hec.ca

Chantale Mailhot est professeure adjointe au département de l'enseignement du management de HEC Montréal. Docteure en management (Ph.D.) de cette même école, elle a fait des études postdoctorales dans le domaine de la gestion de l'innovation dans un laboratoire de recherche de l'Université Louis Pasteur à Strasbourg. Ses travaux portent sur les pratiques de gestion et de coordination dans les nouvelles formes d'organisation telles que les partenariats de recherche entre l'entreprise et l'université. Elle s'intéresse aussi aux liens entre la recherche, l'enseignement et les enjeux actuels que vivent des praticiens de la gestion.

Patrick Pelletier est détenteur d'une maîtrise en sociologie de l'Université du Québec à Montréal et termine actuellement sa thèse de doctorat en management à HEC Montréal. Ses intérêts de recherche portent spécifiquement sur le changement institutionnel en contexte universitaire sous les angles de l'innovation pédagogique, de l'intégration des technologies vouées à l'apprentissage, des politiques de l'État en matière d'enseignement supérieur et de la collaboration entre école et entreprise. 
Véronique Schaeffer est docteure en Sciences de Gestion et maître de conférences à l'Université Louis Pasteur de Strasbourg. Elle exerce ses activités de recherche au sein du BETA. Ses travaux portent sur les défis posés au management des universités par l'évolution de leur rôle dans la société. Plus spécifiquement elle s'intéresse aux stratégies de développement de la valorisation de la recherche universitaire et à la spécificité de la démarche stratégique au sein des universités. Ses travaux s'inscrivent également dans les débats actuels sur le rôle des écoles de management dans l'évolution des pratiques de management. 